\title{
Performance of an $M / M / 1$ Retrial Queue with Working Vacation Interruption and Classical Retrial Policy
}

\author{
Tao Li, ${ }^{1}$ Liyuan Zhang, ${ }^{2}$ and Shan Gao ${ }^{3}$ \\ ${ }^{1}$ School of Science, Shandong University of Technology, Zibo 255049, China \\ ${ }^{2}$ School of Business, Shandong University of Technology, Zibo 255049, China \\ ${ }^{3}$ School of Mathematics and Statistics, Fuyang Normal College, Fuyang 236037, China \\ Correspondence should be addressed to Tao Li; liltaot@126.com
}

Received 8 December 2015; Revised 30 April 2016; Accepted 5 May 2016

Academic Editor: Yi-Kuei Lin

Copyright (C) 2016 Tao Li et al. This is an open access article distributed under the Creative Commons Attribution License, which permits unrestricted use, distribution, and reproduction in any medium, provided the original work is properly cited.

\begin{abstract}
An $\mathrm{M} / \mathrm{M} / 1$ retrial queue with working vacation interruption is considered. Upon the arrival of a customer, if the server is busy, it would join the orbit of infinite size. The customers in the orbit will try for service one by one when the server is idle under the classical retrial policy with retrial rate $n \alpha$, where $n$ is the size of the orbit. During a working vacation period, if there are customers in the system at a service completion instant, the vacation will be interrupted. Under the stable condition, the probability generating functions of the number of customers in the orbit are obtained. Various system performance measures are also developed. Finally, some numerical examples and cost optimization analysis are presented.
\end{abstract}

\section{Introduction}

Queueing models with server vacations have been well studied in the past three decades and successfully applied in manufacturing and production systems, service systems, and communication systems. Some vacation queues can be found in Tian and Zhang [1]. On the basis of ordinary vacation, Servi and Finn [2] first introduced a class of semivacation policy known as working vacation (WV), where the server provides service at a lower speed during the vacation period rather than stopping service completely. The motivation of analyzing $\mathrm{M} / \mathrm{M} / \mathrm{l} / \mathrm{WV}$ queue is to model approximately a multiqueue system where each queue can be served at one of two service rates. From then onwards, several works [3-5] have appeared that analyzed the single server queue with working vacations. In order to utilize the server effectively, $\mathrm{Li}$ and Tian [6] introduced vacation interruption policy. During the working vacation period, if at least one customer is present in the system at a service completion epoch, the server will interrupt the vacation and resume regular service. Working vacation interruption has become an important aspect. Using the matrix analytic method, Li and Tian [7] analyzed a GI/Geo/1 queue with working vacations and vacation interruption and $\mathrm{Li}$ et al. [8] discussed the GI/M/1 queue. Using the method of a supplementary variable, Zhang and Hou [9] investigated an $\mathrm{M} / \mathrm{G} / 1$ queue with working vacations and vacation interruption.

Retrial queueing systems are described by the feature that the arriving customers who find the server busy join the retrial orbit to try again for their requests. During the last two decades, retrial queues have been studied extensively because of their applications in telephone switching systems, telecommunication networks, and computer systems. Readers can refer to Artalejo and Gómez-Corral [10] and Choudhury et al. [11]. Recently, the retrial queueing systems with working vacations have been investigated extensively. Do [12] first studied an $\mathrm{M} / \mathrm{M} / 1$ retrial queue with working vacations. $\mathrm{Li}$ et al. [13] considered a discrete time $\mathrm{Geo} / \mathrm{Geo} / 1$ retrial queue with working vacations and vacation interruption and Liu and Song [14] introduced nonpersistent customers into the $\mathrm{Geo} / \mathrm{Geo} / 1$ retrial queue with working vacations. Tao et al. 
[15] discussed an $M / M / 1$ retrial queue with collisions and working vacation interruption under N-policy. Using the matrix analytic method, Do [12], Li et al. [13], Liu and Song [14], and Tao et al. [15] obtained the stationary probability distribution and showed the conditional stochastic decomposition for the queue length. Using the method of a supplementary variable, Aissani et al. [16] and Jailaxmi et al. [17] both generalized the model of [12] to an M/G/1 queue. Gao and Wang [18] analyzed a Geo ${ }^{X} / \mathrm{G} / 1$ retrial queue with general retrial times and working vacation interruption, and the continuous-time M/G/1 queue was investigated by Gao et al. [19]. Note that the retrial policy of the above papers is either constant retrial policy or general retrial policy, where only the customer at the head of the orbit can request a service.

Many of the queueing systems with repeated attempts operate under the classical retrial policy, where each block of customers generate a stream of repeated attempts independently of the rest of the customers in the orbit. In telephone systems, the calls which will be reattempted can be modeled by an infinite server queue where the time until reattempt follows an exponential distribution. Thus, it needs to discuss a retrial queue with working vacation under the classical retrial policy. Ayyappan et al. [20] first studied such a queueing system. In order to obtain the steady state probability vector, the orbit size is restricted to $M$ such that any arriving customer finding the orbit full is considered lost. In this paper, we also analyze an $M / M / 1$ retrial queue with multiple working vacations under the classical retrial policy, and vacation interruption policy is considered. Ayyappan et al. [20] mainly focus on numerical computation and approximation, and they apply the direct truncation method to find the steady state probability vector. We place more emphasis on the analytic solutions, and the orbit size is always infinite in our model. The novelty of this investigation is that we use probability generating function method to deal with the steady state joint distribution of the server state and the number of customers in the orbit, and the ergodicity condition is obtained by Foster's criterion. Finally, a cost minimization problem is discussed.

This paper is organized as follows. In Section 2, we establish the model. Using Foster's criterion, the stable condition is obtained. In Section 3, we get the balance equations and derive the probability generating function of the number of customers in the orbit. Various performance measures of this model are discussed in Section 4. Section 5 presents some numerical examples and cost optimization analysis. Finally, Section 6 concludes the paper.

\section{Model Formulation and Stable Condition}

Let us consider an $\mathrm{M} / \mathrm{M} / 1$ retrial queue with working vacations and vacation interruption. Customers arrive according to a Poisson process with rate $\lambda$. Upon the arrival of customers, if the server is free, arriving customers get service immediately. If the server is occupied, customers are forced to wait in the orbit of infinite size. In this paper, we adopt the classical retrial policy with retrial rate $n \alpha$, where $n$ is the orbit size. Upon the arrival of retrials, if the server is busy, retrials will go back to the orbit. If the server is not occupied, arriving retrials get services immediately. The service rate is $\mu$ when the system is not on vacation. The single server takes a working vacation when the system becomes empty. Vacation time follows an exponential distribution with parameter $\theta$. During the vacation period, customers can be served with rate $\eta$. At a service completion instant in the vacation period, if there are customers in the system at that moment, the vacation is interrupted and the server comes back to the normal working level. The working vacation will continue if the system is empty. At the end of each vacation, the server only takes another new vacation if the system is empty.

Next, we give an application example of this model. In our daily life, some social organizations can provide telephone psychological counseling. Here, we consider a telephone counseling system staffed with a psychological consultant (called main server) and an assistant (called substitute server). The assistant only provides service to people (called customers) when the consultant does not work. Generally speaking, there is a telephone operator who is responsible for establishing communications between servers and customers; the operator also needs to note down the calls' information in a registration form (corresponding to the "orbit"). When a person makes a call and if the server is free, the operator notes down the information and the customer is served immediately by the consultant or the assistant. If the server is busy, on the other hand, the operator tells the customer to call again some time later (called retrial), and the customer's information should also be noted down. When a retrial customer makes a call again, the operator does not need to note down the information again. Moreover, if the customer in the registration form completes his service, the telephone operator will make a note; then he can know whether the customer completed his service or not. When the operator finds that all customers in the registration form have completed their services (i.e., there is no customer in the orbit and the system is empty), the consultant will rest from his work (begin a vacation). During the consultant's vacation period, the assistant will provide service. If a service is completed by the assistant and if there are customers in the registration form who have not received services (i.e., there are customers in the orbit and the system is nonempty), the consultant will come back from his vacation no matter whether his vacation ends or not (vacation interruption happens). Furthermore, when a vacation ends and if all customers in the registration form have completed their services, the consultant begins another vacation. Otherwise, the consultant takes over the assistant. In order to understand the customer's need, the consultant will restart service no matter how long the assistant has served.

Let $Q(t)$ be the number of customers in the orbit at time $t$ and let $J(t)$ be the state of server at time $t$. There are four possible states of the server as follows: 
$J(t)= \begin{cases}0, & \text { the server is in a working vacation period at time } t \text { and the server is free, } \\ 1, & \text { the server is in a working vacation period at time } t \text { and the server is busy, } \\ 2, & \text { the server is during a normal service period at time } t \text { and the server is free, } \\ 3, & \text { the server is during a normal service period at time } t \text { and the server is busy. }\end{cases}$

Note that when the orbit is empty, it is impossible that the server is free in a normal service period. Thus, $\{Q(t), J(t)\}$ is a Markov process with state space

$$
\Omega=\{(0, j), \quad j=0,1,3\} \cup\{(k, j), k \geq 1, j=1,2,3\}
$$

Remark 1. Note that, in this model, we consider working vacation interruption policy, so the state $\{(k, 0), k \geq 1\}$ does not exist.

Define

$$
\begin{aligned}
& a_{k}=\int_{0}^{\infty} \frac{(\lambda x)^{k}}{k !} e^{-\lambda x} \mu e^{-\mu x} d x, \\
& b_{k}=\int_{0}^{\infty} \frac{(\lambda x)^{k}}{k !} e^{-\lambda x} e^{-\theta x} \eta e^{-\eta x} d x, \\
& c_{k}=\int_{0}^{\infty} \frac{(\lambda x)^{k}}{k !} e^{-\lambda x} \theta e^{-\theta x} e^{-\eta x} d x,
\end{aligned}
$$

where $\left\{a_{k}, k \geq 0\right\}$ is the probability that $k$ customers arrive during a normal service time; $\left\{b_{k}, k \geq 0\right\}$ represents the probability that $k$ customers arrive during a lower service time and the working vacation does not end; $\left\{c_{k}, k \geq 0\right\}$ explains the probability that $k$ customers arrive before the working vacation ends and no service is completed.

Let $\left\{t_{n} ; n=1,2, \ldots\right\}$ be the sequence of epochs at which a normal service or a lower service completion occurs and $Q_{n}=Q\left(t_{n}^{+}\right)$; then the sequence of random variables $\left\{Q_{n} ; n=\right.$ $1,2, \ldots\}$ forms an embedded Markov chain.

Theorem 2. The embedded Markov chain $\left\{Q_{n} ; n=1,2, \ldots\right\}$ is ergodic if and only if $\rho=\lambda / \mu<1$.

Proof. It is not difficult to see that $\left\{Q_{n} ; n=1,2, \ldots\right\}$ is an irreducible and aperiodic Markov chain, and the transition probability is given by

$$
P=\left(\begin{array}{ccccc}
P_{00} & P_{01} & P_{02} & P_{03} & \cdots \\
P_{10} & P_{11} & P_{12} & P_{13} & \cdots \\
0 & P_{21} & P_{22} & P_{23} & \cdots \\
0 & 0 & P_{32} & P_{33} & \cdots \\
\vdots & \vdots & \vdots & \vdots & \ddots
\end{array}\right)
$$

where

$$
\begin{aligned}
& P_{n k} \\
& \quad= \begin{cases}b_{k}+\sum_{j=0}^{k} c_{j} a_{k-j}, & n=0, k \geq 0, \\
\frac{n \alpha}{n \alpha+\lambda} a_{0}, & n \geq 1, k=n-1, \\
\frac{n \alpha}{n \alpha+\lambda} a_{k+1-n}+\frac{\lambda}{n \alpha+\lambda} a_{k-n}, & n \geq 1, k \geq n .\end{cases}
\end{aligned}
$$

In order to discuss the ergodicity of the chain $\left\{Q_{n} ; n=\right.$ $1,2, \ldots\}$, we use Foster's criterion with the mean drift

$$
x_{n}=E\left[f\left(Q_{k+1}\right)-f\left(Q_{k}\right) \mid Q_{k}=n\right] \text {, }
$$

where the test function $f(n)=n$. From the transition matrix, it can be easily proved that

$$
x_{n}= \begin{cases}\rho \frac{\theta}{\theta+\eta}+\frac{\lambda}{\theta+\eta}, & n=0, \\ \rho-\frac{n \alpha}{n \alpha+\lambda}, & n \geq 1 .\end{cases}
$$

As $n \rightarrow \infty$, there exists $x=\lim _{n \rightarrow \infty} x_{n}=\rho-1$. Applying Foster's criterion, we can get that the embedded Markov chain is ergodic if $\rho<1$. Besides, since $Q_{n+1}-Q_{n} \geq-1$, applying the criterion from Sennott et al. [21], we can obtain that $\rho<1$ is necessary for ergodicity.

Since the arrival process is Poisson, using PASTA property, it can be shown from Burke's theorem (see [22, pp. 187$188])$ that the steady state probabilities of the Markov process $\{Q(t), J(t)\}$ exist if and only if the stable condition $\rho<1$ holds. Now we define the limiting probability

$$
\begin{aligned}
\pi_{k j} & =P\{Q=k, J=j\} \\
& =\lim _{t \rightarrow \infty} P\{Q(t)=k, J(t)=j\}, \quad(k, j) \in \Omega .
\end{aligned}
$$

\section{Balance Equations and Probability Generating Functions}

Figure 1 presents the state transition rate diagram of the system. Under the stable condition $\rho<1$, the set of balance equations is given as follows: 


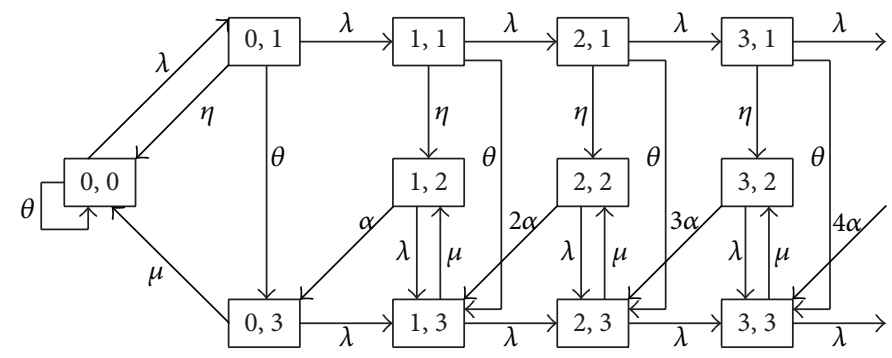

FIGURE 1: State transition rate diagram of the system.

$$
\begin{aligned}
\lambda \pi_{00} & =\eta \pi_{01}+\mu \pi_{03}, \\
(\lambda+\theta+\eta) \pi_{01} & =\lambda \pi_{00}, \\
(\lambda+\mu) \pi_{03}= & \alpha \pi_{12}+\theta \pi_{01}, \\
(\lambda+\theta+\eta) \pi_{n 1}= & \lambda \pi_{n-1,1}, \quad n \geq 1, \\
(\lambda+n \alpha) \pi_{n 2}= & \eta \pi_{n 1}+\mu \pi_{n 3}, \quad n \geq 1, \\
(\lambda+\mu) \pi_{n 3}= & \lambda \pi_{n 2}+\lambda \pi_{n-1,3}+(n+1) \alpha \pi_{n+1,2} \\
& +\theta \pi_{n 1}, \quad n \geq 1 .
\end{aligned}
$$

Define the probability generating functions (pgfs)

$$
\begin{aligned}
& G_{1}(z)=\sum_{n=0}^{\infty} \pi_{n 1} z^{n}, \\
& G_{2}(z)=\sum_{n=1}^{\infty} \pi_{n 2} z^{n}, \\
& G_{3}(z)=\sum_{n=0}^{\infty} \pi_{n 3} z^{n},
\end{aligned}
$$

with $G_{i}^{\prime}(z)=\sum_{n=1}^{\infty} n \pi_{n i} z^{n-1}, i=1,2,3$.

Multiplying by the appropriate power of $z^{n}$ in (10) and (12), summing over $n$, and rearranging the terms, we get

$$
(\lambda+\theta+\eta-\lambda z) G_{1}(z)=\lambda \pi_{00} .
$$

Multiplying by the appropriate power of $z^{n}$ in (13), summing over $n$, and using (9), we obtain

$$
\begin{aligned}
\lambda G_{2}(z)+\alpha z G_{2}^{\prime}(z)= & \eta G_{1}(z)+\mu G_{3}(z)-\eta \pi_{01} \\
& -\mu \pi_{03} \\
= & \eta G_{1}(z)+\mu G_{3}(z)-\lambda \pi_{00} .
\end{aligned}
$$

In a similar way, from (11) and (14), we derive

$$
(\lambda+\mu-\lambda z) G_{3}(z)=\lambda G_{2}(z)+\alpha G_{2}^{\prime}(z)+\theta G_{1}(z) .
$$

From (16), we get

$$
G_{1}(z)=\frac{\lambda}{\lambda+\theta+\eta-\lambda z} \pi_{00} .
$$

From (17), we obtain

$$
G_{3}(z)=\frac{\lambda}{\mu} G_{2}(z)+\frac{\alpha z}{\mu} G_{2}^{\prime}(z)-\frac{\eta}{\mu} G_{1}(z)+\frac{\lambda}{\mu} \pi_{00} .
$$

Taking (20) into (18), using (19), after some computation, we can derive

$$
\begin{aligned}
G_{2}^{\prime}(z) & -\frac{\lambda^{2}}{(\mu-\lambda z) \alpha} G_{2}(z) \\
= & \frac{\lambda^{2} \pi_{00}}{(\mu-\lambda z)(1-z) \alpha}+\frac{\lambda \pi_{00}}{(1-z) \alpha} \\
& -\frac{\eta \lambda \pi_{00}}{(\lambda+\theta+\eta-\lambda z)(1-z) \alpha} \\
& -\frac{(\theta \mu+\lambda \eta) \lambda \pi_{00}}{(\lambda+\theta+\eta-\lambda z)(\mu-\lambda z)(1-z) \alpha} .
\end{aligned}
$$

Solving the first-order differential equation, we have

$$
\begin{gathered}
G_{2}(z)=(\mu-\lambda z)^{-\lambda / \alpha}\left[\frac{\lambda^{2} \pi_{00}}{\alpha} K_{1}(z)+\frac{\lambda \pi_{00}}{\alpha} K_{2}(z)\right. \\
\left.-\frac{\lambda \eta \pi_{00}}{\alpha} K_{3}(z)-\frac{(\theta \mu+\lambda \eta) \lambda \pi_{00}}{\alpha} K_{4}(z)\right]
\end{gathered}
$$

where

$$
\begin{aligned}
& K_{1}(z)=\int_{0}^{z}(1-x)^{-1}(\mu-\lambda x)^{\lambda / \alpha-1} d x, \\
& K_{2}(z)=\int_{0}^{z}(1-x)^{-1}(\mu-\lambda x)^{\lambda / \alpha} d x, \\
& K_{3}(z) \\
& =\int_{0}^{z}(\lambda+\theta+\eta-\lambda x)^{-1}(1-x)^{-1}(\mu-\lambda x)^{\lambda / \alpha} d x, \\
& K_{4}(z) \\
& =\int_{0}^{z}(\lambda+\theta+\eta-\lambda x)^{-1}(1-x)^{-1}(\mu-\lambda x)^{\lambda / \alpha-1} d x .
\end{aligned}
$$

From (17) and (18), substituting $G_{2}^{\prime}(z)$, we get

$$
G_{3}(z)=\frac{\lambda(1-z) G_{2}(z)-(\eta+\theta z) G_{1}(z)+\lambda \pi_{00}}{(\mu-\lambda z)(1-z)} .
$$


From (19), (22), and (24), we can see that $G_{1}(z), G_{2}(z)$, and $G_{3}(z)$ are expressed in terms of $\pi_{00}$. In the next section, using normalization condition, we will obtain $\pi_{00}$.

\section{Performance Measures}

From (19), we have

$$
\begin{aligned}
G_{1}(1) & =\frac{\lambda}{\theta+\eta} \pi_{00}, \\
G_{1}^{\prime}(1) & =\lim _{z \rightarrow 1} G_{1}^{\prime}(z)=\lim _{z \rightarrow 1} \frac{\lambda^{2}}{(\lambda+\theta+\eta-\lambda z)^{2}} \pi_{00} \\
& =\frac{\lambda^{2}}{(\theta+\eta)^{2}} \pi_{00} .
\end{aligned}
$$

From (22), we get

$$
G_{2}(1)=K \pi_{00}
$$

where

$$
\begin{aligned}
K & =(\mu-\lambda)^{-\lambda / \alpha}\left[\frac{\lambda^{2}}{\alpha} K_{1}(1)+\frac{\lambda}{\alpha} K_{2}(1)-\frac{\lambda \eta}{\alpha} K_{3}(1)\right. \\
& \left.-\frac{(\theta \mu+\lambda \eta) \lambda}{\alpha} K_{4}(1)\right] .
\end{aligned}
$$

From (24), using L'Hospital rule, we obtain

$$
\begin{aligned}
& G_{3}(1)=\lim _{z \rightarrow 1} G_{3}(z) \\
& =\lim _{z \rightarrow 1} \frac{-\lambda G_{2}(z)+\lambda(1-z) G_{2}^{\prime}(z)-\theta G_{1}(z)-(\eta+\theta z) G_{1}^{\prime}(z)}{-\lambda(1-z)-(\mu-\lambda z)}
\end{aligned}
$$

$$
\begin{aligned}
& =\frac{\lambda G_{2}(1)+\theta G_{1}(1)+(\eta+\theta) G_{1}^{\prime}(1)}{\mu-\lambda} \\
& =\frac{\lambda K(\theta+\eta)+\lambda \theta+\lambda^{2}}{(\mu-\lambda)(\theta+\eta)} \pi_{00} .
\end{aligned}
$$

Since

$$
\pi_{00}+G_{1}(1)+G_{2}(1)+G_{3}(1)=1,
$$

taking (25), (27), and (29) into the above equation, we can derive

$$
\begin{aligned}
\pi_{00} & =\left[1+\frac{\lambda}{\theta+\eta}+K+\frac{\lambda K(\theta+\eta)+\lambda \theta+\lambda^{2}}{(\mu-\lambda)(\theta+\eta)}\right]^{-1} \\
& =\left[\frac{\lambda(\mu-\eta)+\mu(1+K)(\theta+\eta)}{(\mu-\lambda)(\theta+\eta)}\right]^{-1} .
\end{aligned}
$$

Thus, $\pi_{00}$ is found out. From (19), (22), and (24), $G_{1}(z)$, $G_{2}(z)$, and $G_{3}(z)$ are completely computed. And the pgf of the number of customers in the orbit is given by

$$
G(z)=\pi_{00}+G_{1}(z)+G_{2}(z)+G_{3}(z) .
$$

The pgf of the number of customers in the system is given by

$$
\widetilde{G}(z)=\pi_{00}+z G_{1}(z)+G_{2}(z)+z G_{3}(z) .
$$

Let $E\left[L_{j}\right]$ denote the average number of customers in the orbit when the server's state is $j, j=1,2,3$.

Differentiating (19) with respect to $z$, we have

$$
E\left[L_{1}\right]=\lim _{z \rightarrow 1} G_{1}^{\prime}(z)=\frac{\lambda^{2}}{(\theta+\eta)^{2}} \pi_{00}
$$

From (18), we can get

$$
E\left[L_{2}\right]=\lim _{z \rightarrow 1} G_{2}^{\prime}(z)=\frac{\mu}{\alpha} G_{3}(1)-\frac{\lambda}{\alpha} G_{2}(1)-\frac{\theta}{\alpha} G_{1}(1) .
$$

Differentiating (24) with respect to $z$ and using L'Hospital rule twice, the expression for $E\left[L_{3}\right]$ is obtained as

$$
E\left[L_{3}\right]=\lim _{z \rightarrow 1} G_{3}^{\prime}(z)=\frac{(\mu-\lambda)(\theta+\eta) G_{1}^{\prime \prime}(1)+2(\theta \mu+\lambda \eta) E\left[L_{1}\right]+2 \lambda(\mu-\lambda) E\left[L_{2}\right]+2 \lambda\left[\theta G_{1}(1)+\lambda G_{2}(1)\right]}{2(\mu-\lambda)^{2}}
$$

and differentiating (19) twice with respect to $z$ and taking $z=$ 1 yield $G_{1}^{\prime \prime}(1)$ as

$$
G_{1}^{\prime \prime}(1)=\frac{2 \lambda^{3}}{(\theta+\eta)^{3}} \pi_{00}
$$

Therefore, the average orbit length $(E[L])$ is given by

$$
E[L]=\lim _{z \rightarrow 1} G^{\prime}(z)=E\left[L_{1}\right]+E\left[L_{2}\right]+E\left[L_{3}\right] .
$$

And the average system length $(E[\widetilde{L}])$ is derived as

$$
E[\widetilde{L}]=\lim _{z \rightarrow 1} \widetilde{G^{\prime}}(z)=E[L]+G_{1}(1)+G_{3}(1) .
$$

The probability that the server is busy is

$$
P_{b}=P\{J=1\}+P\{J=3\}=G_{1}(1)+G_{3}(1) .
$$


The probability that the server is free is

$$
P_{f}=P\{J=0\}+P\{J=2\}=\pi_{00}+G_{2}(1)=1-P_{b} .
$$

The probability that the server is in a working vacation period is given by

$$
P_{w}=P\{J=0\}+P\{J=1\}=\pi_{00}+G_{1}(1) .
$$

The probability that the server is in a normal service period is given by

$$
\begin{aligned}
P_{n} & =P\{J=2\}+P\{J=3\}=G_{1}(2)+G_{2}(3) \\
& =1-P_{w} .
\end{aligned}
$$

Let $E[W](E[\widetilde{W}])$ be the expected waiting (sojourn) time of a customer in the orbit (system), using Little's formula:

$$
\begin{aligned}
& E[W]=\frac{E[L]}{\lambda}, \\
& E[\widetilde{W}]=\frac{E[\widetilde{L}]}{\lambda} .
\end{aligned}
$$

The system busy period $T$ is defined as the period that starts at an epoch when an arriving customer finds an empty system and ends at the departure epoch at which the system is empty. Using the theory of regenerative process, we get

$$
\pi_{00}=\frac{E\left[T_{00}\right]}{E\left[T_{00}\right]+E[T]},
$$

where $T_{00}$ is the time length that the system is in the state $(0,0)$. Since the interarrival time between two customers follows exponential distribution with parameter $\lambda$, we have $E\left[T_{00}\right]=1 / \lambda$. Thus, $E[T]=\lambda^{-1}\left(\pi_{00}^{-1}-1\right)$.

\section{Numerical Results}

In this section, under the stable condition, we present some numerical examples to illustrate the effect of the varying parameters on the mean orbit length $E[L]$, the probability that the system is busy $1-\pi_{0,0}$, and the probabilities of server's state $\left(P_{b}, P_{f}, P_{w}\right.$, and $\left.P_{n}\right)$. Moreover, a cost minimization problem is also discussed. The various parameters of this model are arbitrarily chosen as $\lambda=2, \eta=1, \mu=5, \alpha=3$, and $\theta=0.2$, unless they are considered as variables or their values are mentioned in Figures 2, 3, 4, and 5.

5.1. Sensitivity Analysis. Figures 2 and 3 illustrate the effect of $\theta$ on $E[L]$ and $1-\pi_{0,0}$ for different values of $\eta$, respectively. When $\eta<\mu$, we find that $E[L]$ and $1-\pi_{0,0}$ both decrease as $\theta$ increases, and $\theta$ has a noticeable effect on the performance measures which cannot be ignored. An especial case is $\eta=\mu$; that is, the service rate in the vacation period is equal to the service rate in the normal period, and the model we consider reduces to an $\mathrm{M} / \mathrm{M} / 1$ retrial queue with classical retrial policy. Clearly, $\theta$ has no effect on $E[L]$ and $1-\pi_{0,0}$, which agrees with the intuitive expectation.

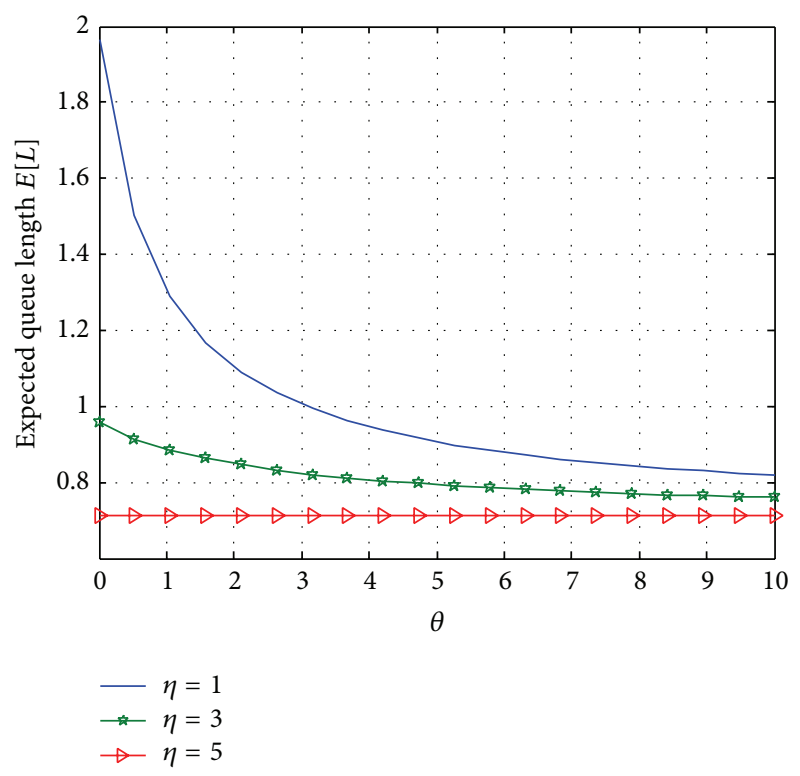

Figure 2: The effect of $\theta$ on $E[L]$ for different values of $\eta$.

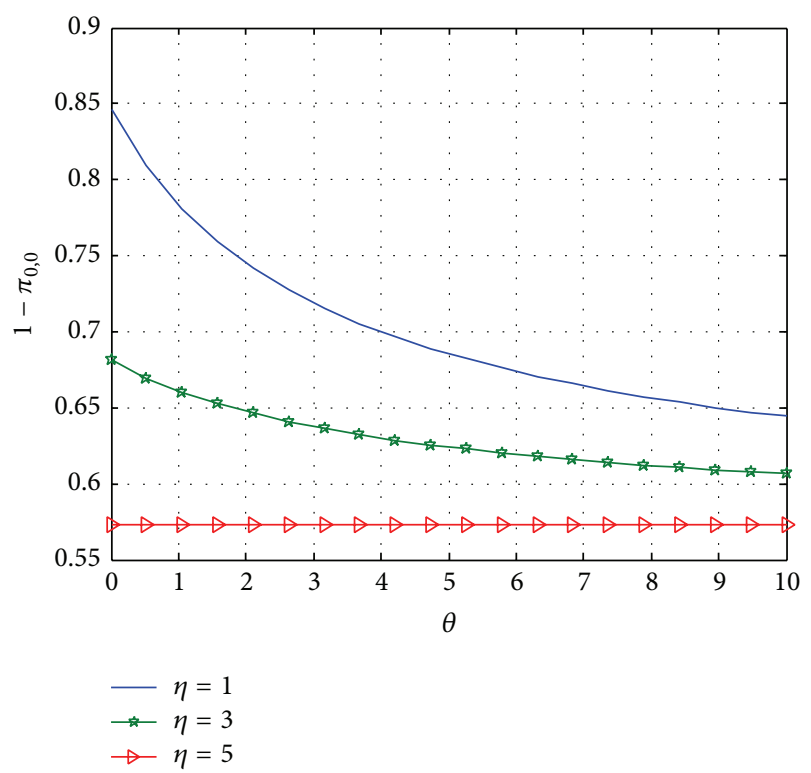

FIgURE 3: The effect of $\theta$ on $1-\pi_{0,0}$ for different values of $\eta$.

The effect of $\eta$ on $E[L]$ is presented in Figure 4; as expected, $E[L]$ decreases with increasing values of $\eta$. When $\eta$ is large, it can be observed that the effect of $\eta$ on $E[L]$ is not obvious as $\eta$ increases; this is because we consider working vacation interruption policy. If the system is not empty at a service completion instant in the vacation period, the server will come back to the normal working level. Moreover, since constant retrial policy means that only the customer at the head of the orbit can request a service, we can find that $E[L]$ of $\mathrm{M} / \mathrm{M} / 1$ queue with classical retrial policy is smaller than that of $\mathrm{M} / \mathrm{M} / 1$ queue with constant retrial policy. As shown in Figure $5, P_{b}$ decreases with increasing values of $\eta$, and $P_{f}$ has the opposite tendency. The reason is that as $\eta$ increases, 


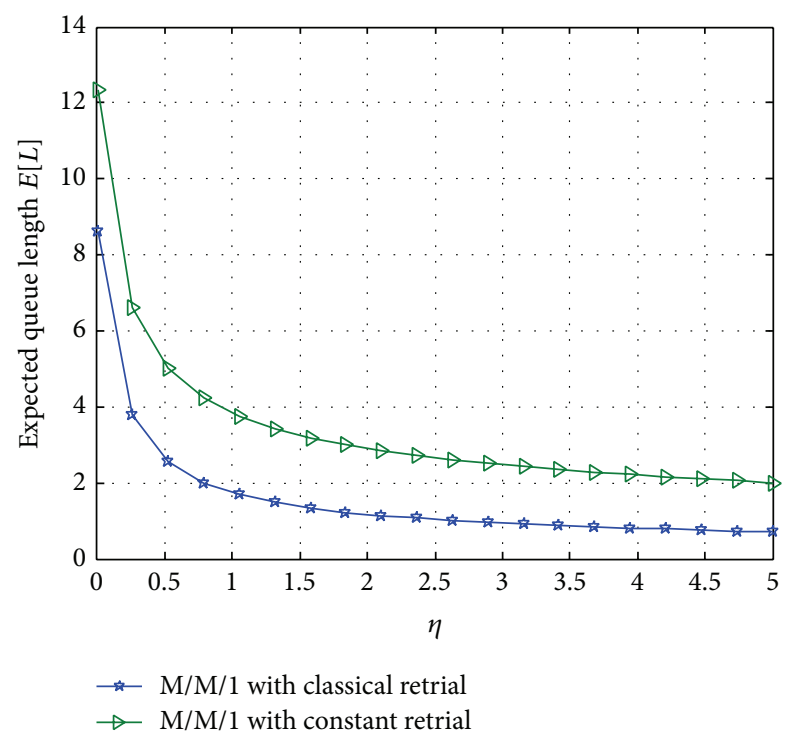

Figure 4: The effect of $\eta$ on $E[L]$ for two different retrial policies.

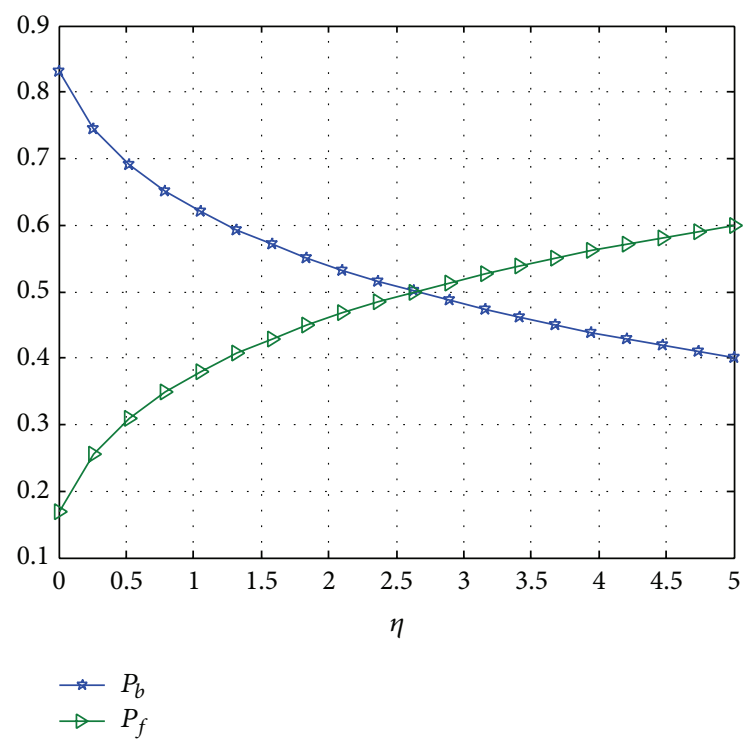

FIgURE 5: The probability of server's state with the change of $\eta$.

the mean orbit length $E[L]$ and the mean service time $1 / \eta$ both decrease.

Figure 6 indicates that $E[L]$ decreases with increasing values of $\alpha$; this is because the mean retrial time decreases as $\alpha$ increases. From the instant when the server becomes idle, an external potential primary customer and retrial customers compete to access the server, and the smaller the mean retrial time is, the bigger the probability that the server is busy is, which leads to decreasing of $E[L]$. Furthermore, under the same condition, decreasing of $E[L]$ results in the increase in the probability that the system is empty, and Figure 7 reveals that $P_{w}$ increases with increasing values of $\alpha$, while $P_{n}$ decreases as $\alpha$ increases. In Figure 6, compared with constant retrial policy, we can also find that classical retrial policy decreases the waiting jobs effectively.

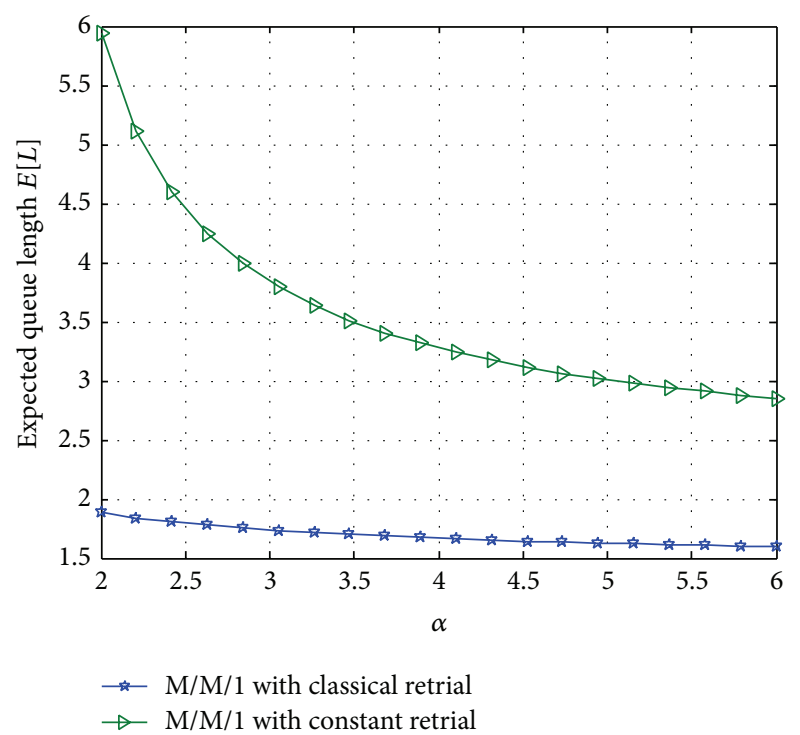

Figure 6: The effect of $\alpha$ on $E[L]$ for two different retrial policies.

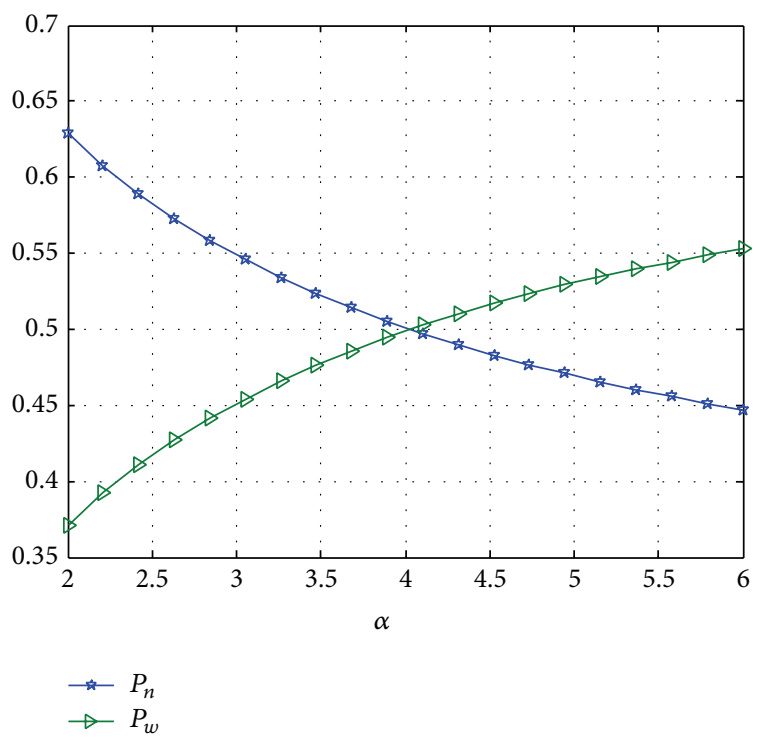

FIgURE 7: The probability of server's state with the change of $\alpha$.

5.2. Cost Analysis. In this subsection, we establish a cost function to search for the optimal service rate $\eta$, so as to minimize the expected operating cost function per unit time.

Define the following cost elements:

$C_{L}$ : cost per unit time for each customer present in the orbit.

$C_{\mu}$ : cost per unit time for service during a normal service period.

$C_{\eta}:$ cost per unit time for service in a working vacation period.

$C_{\theta}$ : fixed cost per unit time during a vacation period. 


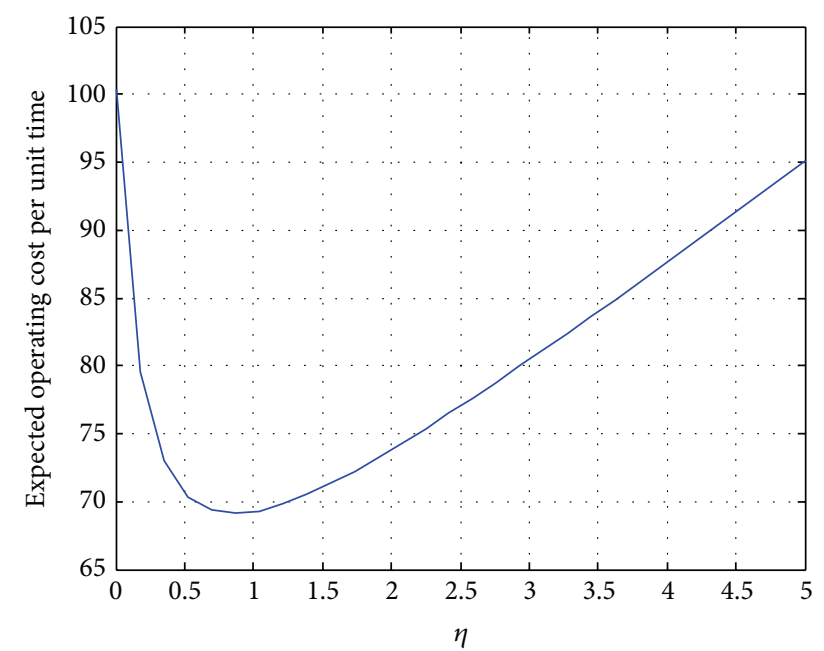

FIGURE 8: Effect of $\eta$ on the expected operating cost per unit time.

Based on the definitions of each cost element listed above, the expected operating cost function per unit time can be given by

$$
\min _{\eta}: f(\eta)=C_{L} E[L]+C_{\mu} \mu+C_{\eta} \eta+C_{\theta} \theta
$$

Because the expected operating cost function is highly nonlinear and complex, it is not easy to get the derivative of it. We assume $C_{L}=6, C_{\mu}=10, C_{\eta}=8$, and $C_{\theta}=4$ and develop approximations by MATLAB program to find the optimum value of $\eta$, say $\eta^{*}$.

From Figure 8, we can see that there is an optimal service rate $\eta$ to minimize the cost. Implementing the computer software MATLAB by the parabolic method and controlling the error by $10^{-4}$, we find the solution $\eta^{*}=0.8584$ with $f\left(\eta^{*}\right)=69.0971$.

\section{Conclusion}

This paper analyzes a single server retrial queue with working vacation interruption under the classical retrial policy. Using embedded Markov chain and Foster's criterion, we get the condition of stability. The pgf of the number of customers in the orbit is obtained, and some important performance measures are also discussed. Moreover, the effects of various parameters on the system performance measures are examined numerically. Under the stable condition, a cost minimization problem is considered. For future research, using the same method, one can investigate a similar model with batch arrival and without vacation interruption.

\section{Competing Interests}

The authors declare that there are no competing interests regarding the publication of this paper.

\section{Acknowledgments}

This work is supported by the National Natural Science Foundation of China (nos. 11301306 and 11401348) and the Natural Science Foundation of Anhui Higher Education Institutions (no. KJ2014ZD21).

\section{References}

[1] N. Tian and Z. G. Zhang, Vacation Queueing Models-Theory and Applications, Springer, New York, NY, USA, 2006.

[2] L. D. Servi and S. G. Finn, "M/M/1 queues with working vacations (M/M/1/WV)," Performance Evaluation, vol. 50, no. 1, pp. 41-52, 2002.

[3] Y. Baba, "Analysis of a GI/M/1 queue with multiple working vacations," Operations Research Letters, vol. 33, no. 2, pp. 201209, 2005.

[4] D.-A. Wu and H. Takagi, "M/G/1 queue with multiple working vacations," Performance Evaluation, vol. 63, no. 7, pp. 654-681, 2006.

[5] J.-H. Li, N.-S. Tian, and W.-Y. Liu, "Discrete-time GI/Geo/1 queue with multiple working vacations," Queueing Systems, vol. 56, no. 1, pp. 53-63, 2007.

[6] J. Li and N. Tian, "The M/M/1 queue with working vacations and vacation interruptions," Journal of Systems Science and Systems Engineering, vol. 16, no. 1, pp. 121-127, 2007.

[7] J.-H. Li and N.-S. Tian, "The discrete-time GI/Geo/1 queue with working vacations and vacation interruption," Applied Mathematics and Computation, vol. 185, no. 1, pp. 1-10, 2007.

[8] J.-H. Li, N.-S. Tian, and Z.-Y. Ma, "Performance analysis of GI/M/1 queue with working vacations and vacation interruption," Applied Mathematical Modelling, vol. 32, no. 12, pp. 27152730, 2008.

[9] M. Zhang and Z. Hou, "Performance analysis of M/G/1 queue with working vacations and vacation interruption," Journal of Computational and Applied Mathematics, vol. 234, no. 10, pp. 2977-2985, 2010.

[10] J. R. Artalejo and A. Gómez-Corral, Retrial Queueing Systems: A Computational Approach, Springer, Berlin, Germany, 2008.

[11] G. Choudhury, L. Tadj, and M. Deka, "An unreliable server retrial queue with two phases of service and general retrial times under Bernoulli vacation schedule," Quality Technology \& Quantitative Management, vol. 12, no. 4, pp. 437-464, 2015.

[12] T. V. Do, "M/M/1 retrial queue with working vacations," Acta Informatica, vol. 47, no. 1, pp. 67-75, 2010.

[13] T. Li, Z. Wang, and Z. Liu, "Geo/Geo/1 retrial queue with working vacations and vacation interruption," Journal of Applied Mathematics and Computing, vol. 39, no. 1-2, pp. 131-143, 2012.

[14] Z. Liu and Y. Song, "Geo/Geo/1 retrial queue with nonpersistent customers and working vacations," Journal of Applied Mathematics and Computing, vol. 42, no. 1-2, pp. 103-115, 2013.

[15] L. Tao, Z. Liu, and Z. Wang, "M/M/1 retrial queue with collisions and working vacation interruption under N-policy," RAIRO Operations Research, vol. 46, no. 4, pp. 355-371, 2012.

[16] A. Aissani, S. Taleb, T. Kernane, G. Saidi, and D. Hamadouche, "An M/G/1 retrial queue with working vacation," in Advances in Systems Science: Proceedings of the International Conference on Systems Science 2013 (ICSS 2013), vol. 240 of Advances in Intelligent Systems and Computing, pp. 443-452, Springer, Berlin, Germany, 2014. 
[17] V. Jailaxmi, R. Arumuganathan, and M. S. Kumar, "Performance analysis of single server non-Markovian retrial queue with working vacation and constant retrial policy," RAIRO Operations Research, vol. 48, no. 3, pp. 381-398, 2014.

[18] S. Gao and J. Wang, "Discrete-time $\mathrm{Geo}^{X} / \mathrm{G} / 1$ retrial queue with general retrial times, working vacations and vacation interruption," Quality Technology \& Quantitative Management, vol. 10, no. 4, pp. 495-512, 2013.

[19] S. Gao, J. Wang, and W. W. Li, "An M/G/1 retrial queue with general retrial times, working vacations and vacation interruption," Asia-Pacific Journal of Operational Research, vol. 31, no. 2, Article ID 1440006, 25 pages, 2014.

[20] G. Ayyappan, A. Ganapathy, and G. Sekar, "Retrial queueing system with single working vacation under pre-emptive priority service," International Journal of Computer Applications, vol. 2, no. 2, pp. 28-35, 2010.

[21] L. I. Sennott, P. A. Humblet, and R. L. Tweedie, "Mean drifts and the nonergodicity of Markov chains," Operations Research, vol. 31, no. 4, pp. 783-789, 1983.

[22] R. B. Cooper, Introduction to Queueing Theory, North-Holland, New York, NY, USA, 1981. 


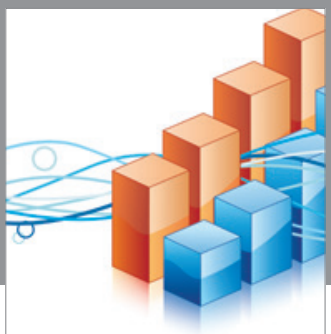

Advances in

Operations Research

vatem alat4

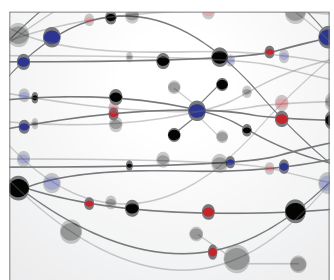

\section{The Scientific} World Journal
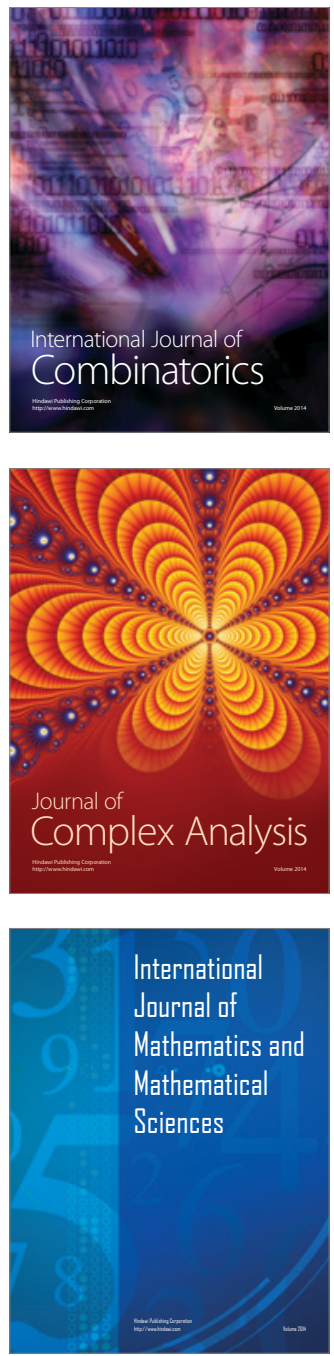
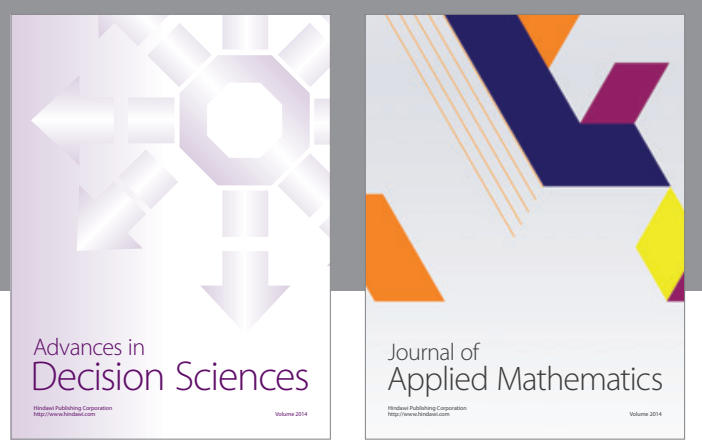

Algebra

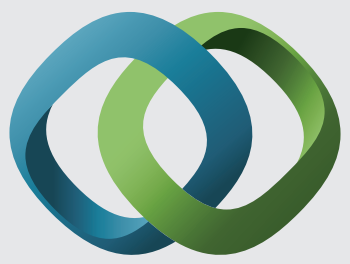

\section{Hindawi}

Submit your manuscripts at

http://www.hindawi.com
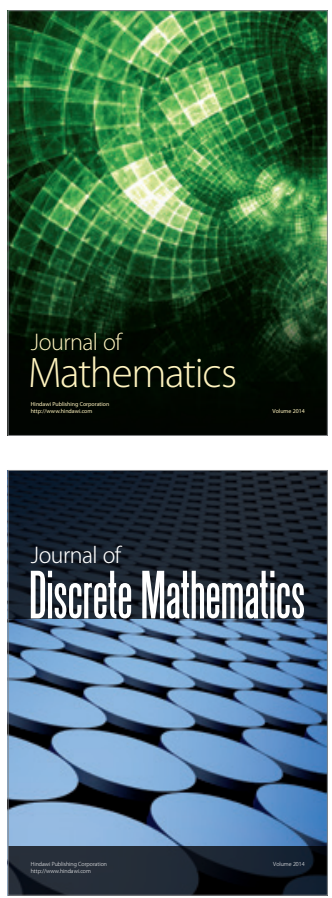

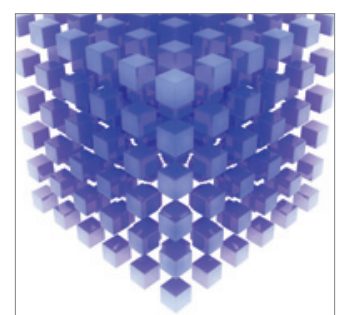

Mathematical Problems in Engineering
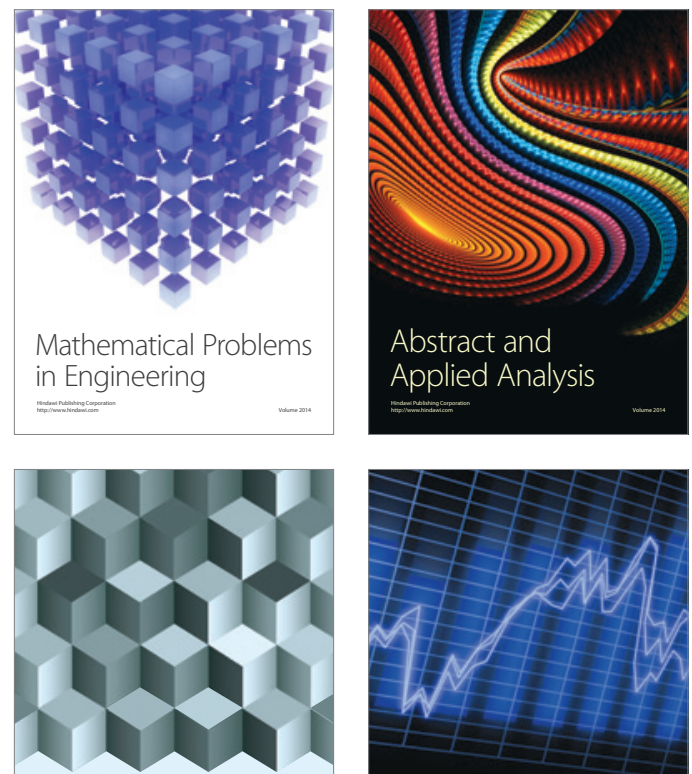

Journal of

Function Spaces

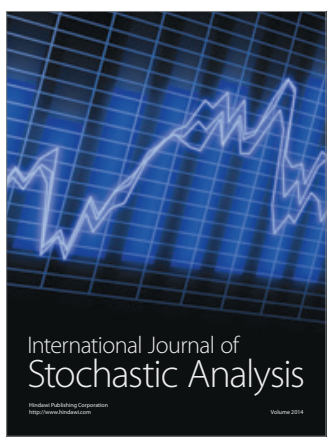

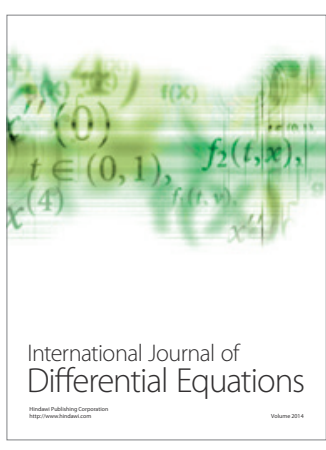
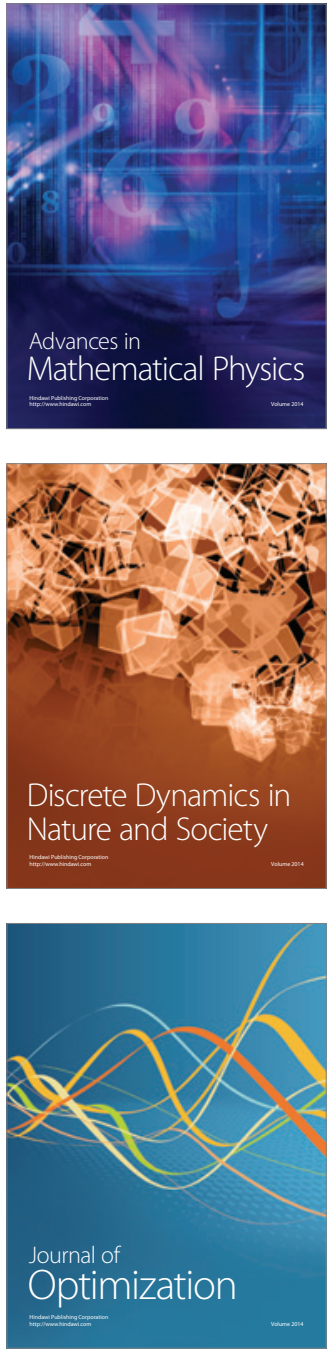